\title{
Noncontact measurement of heart rate using facial video illuminated under natural light and signal weighted analysis
}

\author{
Yonggang Yan ${ }^{\mathrm{a}, \mathrm{b},{ }^{*}}$, Xiang $\mathrm{Ma}^{\mathrm{a}}$, Lifeng Yao and Jianfei Ouyang ${ }^{\mathrm{a}}$ \\ ${ }^{a}$ State Key Laboratory of Precision Measurement Technology and Instruments, Tianjin University, 92 \\ Weijin Road, Nankai District, Tianjin, P.R. China \\ ${ }^{b}$ Precision Engineering Institute, Henan Polytechnic University, 2001 Shiji Road, Shanyang District, \\ Jiaozuo, P.R. China
}

\begin{abstract}
Non-contact and remote measurements of vital physical signals are important for reliable and comfortable physiological self-assessment. We presented a novel optical imaging-based method to measure the vital physical signals. Using a digital camera and ambient light, the cardiovascular pulse waves were extracted better from human color facial videos correctly. And the vital physiological parameters like heart rate were measured using a proposed signal-weighted analysis method. The measured HRs consistent with those measured simultaneously with reference technologies $(r=0.94$, $p<0.001$ for HR). The results show that the imaging-based method is suitable for measuring the physiological parameters, and provide a reliable and comfortable measurement mode. The study lays a physical foundation for measuring multiphysiological parameters of human noninvasively.
\end{abstract}

Keywords: Biomedical engineering, noncontact cardiovascular measurement, heart rate, blood pressure, pulse wave

\section{Introduction}

Estimating human cardiovascular status (CVS) such as heart rate (HR), blood pressure (BP), and breathing pattern are basic requirements in the diagnosis and treatment of various diseases [1-4]. Traditionally, these physiological signals are detected using a contact mode like cuff-oscillometric and electrocardiography (ECG) measurement only in hospital or clinical settings. An important recent trend is the development of non-contact measurement techniques for tracking the vital physiological signals based on optical methods known as photoplethysmography (PPG) that would primary healthcare of common people. The technique of PPG can bring more valuable information for the researchers about the cardiovascular system like HR, BP, arterial blood oxygen saturation, respiration rate, and cardiac autonomic function by detecting the intensity of the output diffuse reflective light or transmission light from the skin $[5,6]$.

\footnotetext{
* Address for correspondence: Yonggang Yan, Precision Engineering Institute, Henan Polytechnic University, 2001 Shiji Road, Shanyang District, Jiaozuo, P.R. China. Tel: +86 391398 7511; Fax: +86 391398 7529; E-mail: tjzyan@tju.edu.cn.
} 
The approaches using the technique can usually be divided into two measurements. The first one is optical single-point detection of the measured subjects pressed against the devices built in the devices to perform PPG [7]. While useful, this approach requires steady physical contact of one's finger or ear with the PPG devices [8], which makes the measured person feel uncomfortable and reduces the measurement accuracy of the physiological signals. With the introduction of CCD camera into clinic imaging monitor and diagnosis system, its successful application promotes the development from single-point detection to imaging measurement of large field of view. The imaging PPG is another optical approach based on noncontact mode [9, 10]. Currently, the non-contact measurements have been forming the development trends of the research and its application. Although the remote and noncontact measurements cannot provide the more details than cardiac electrical conduction that ECG offers, these methods can enable long-term and real-time monitoring of other physiological signals such as heart rate, respiratory rate by acquiring them continuously in an unobtrusive and comfortable way. In addition to heart rates and breathing rates, heart rate variability (HRV) was also analyzed [11, 12] from facial videos. More recently, a near-IR enhanced camera was used to obtain HR from a subject's facial area and breathing rate from the subject's chest area [13].

Currently, the methodologies have been investigated through analyzing the intensity of the diffuse reflection light on human face [14-18] based on the PPG technique. Wu, et al [14] amplified the diffuse reflection light intensity signal and measured the flow of blood as it fills the face using the videos illuminated in natural light. Poh, et al [15] developed a noncontact HR measurement method using an independent component analysis (ICA) that separates a multivariate signal into additive subcomponents by assuming the mutual statistical independence of the non-Gaussian source signals. These efforts helped to minimize unwanted noise in the measured HR signals. In addition, Huang, et al primarily investigated a HR and BP measurement using an optical CMOS imaging device at fingertips [18]. However, it is still much more challenging to measure HR. For example, it is possible for HR measurements to terminate due to the ICA algorithm. And especially, in a typical ambient environment, the useful physiological information is mixed into the noise, particularly noise associated with body movement and imaging devices.

This paper primarily describes a novel method for noncontact measurement of HR signals by maximizing the signals and minimizing the noise due to unwanted movement from color video images recording the intensity of the region of interest on the face. Firstly, our detection method is presented and is used to accomplish the measurements of HR from human facial color video images recorded combining a simply optical camera. Secondly, we demonstrate how this method can validate the accuracy of this approach with a commercial measurement device.

\section{Methods and materials}

\subsection{Study description and experimental setup}

While the visible light beams are used to irradiate human face, the changes of the diffuse reflective light intensity from the skin can bring vital human physical information, and are revealed with facial colors. In the work, we employed a CCD camera (Canon Inc.) as a photoelectric detector to record the color change with videos used for analysis and calculation. The CCD camera was tightly mounted on optical platform, and facial videos of the volunteer were captured in color (24-bit RGB $1280 \times 720$ pixels) at 15 fps (frames per second) and were saved with MOV format in the personal computer. In the work, 15 participants ( 7 males, 8 females) aged 20 35 were enrolled for the in-vitro measurements. 


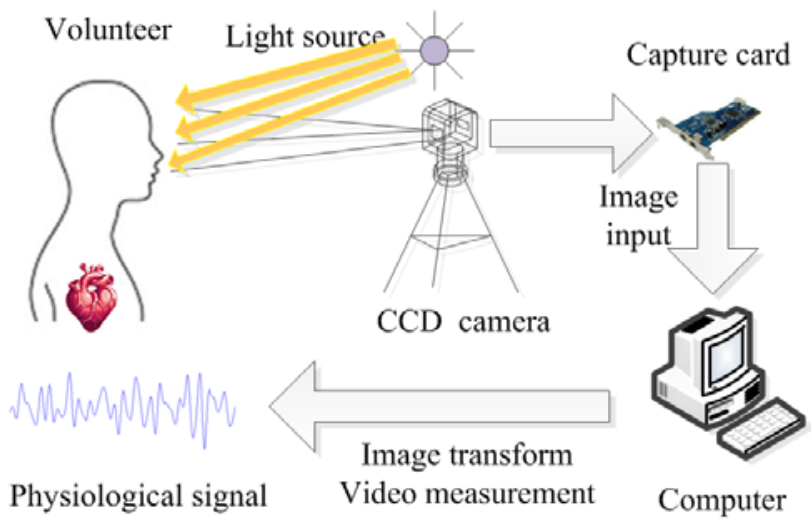

Fig. 1. Schematic illustration of experimental setup.

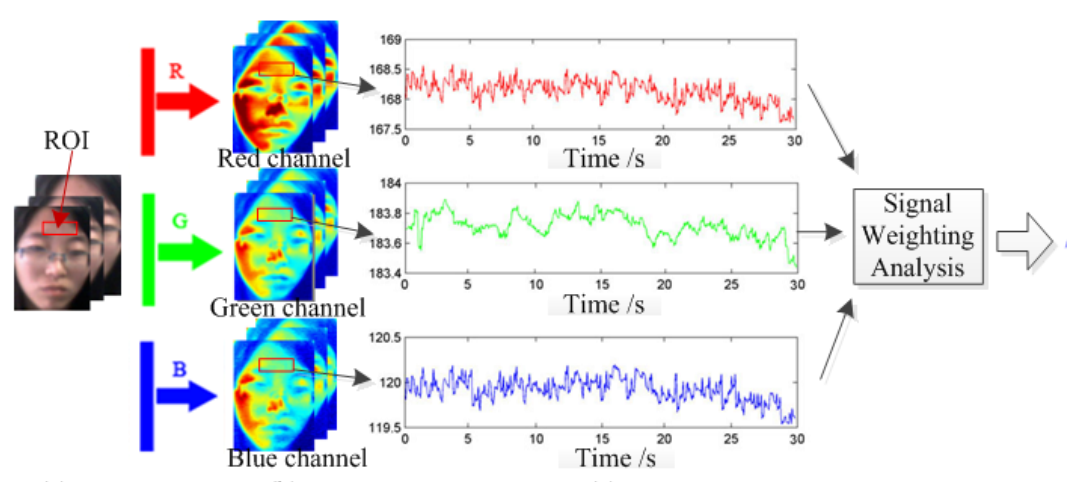

(a)

(b)

(c)

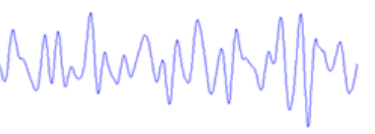

Fig. 2. The proposed methodology. (a) The region of interest (ROI) is selected by hand. (b) The ROI is separated into the three RGB channels and spatially averaged to obtain. (c) The raw RGB traces. (d) The filtered physiological signal using wavelet transform after the raw traces are weighted.

They were comprised of two genders, different ages and different skin colors (Yellows, Whites and Blacks). The experiments were conducted with a plane light as the only source of illumination indoors. A schematic illustration of the experimental setup is shown in Figure 1.

During the video recording, the volunteers were seated in front of an optical platform at a distance of approximately $0.5 \mathrm{~m}$ from the camera, and were not permitted to move anyway. The recording time length was 30 seconds for validation of physiological parameters. In our experiments, an AAMIcertificated and commercial electronic sphygmomanometer (OMRON Corp.) was used to measure the participant's HR for comparison with the proposed method in the paper. Post-analysis and processing of video recordings and physical signals were carried out with custom software by programming in MATLAB (The MathWorks, Inc.).

\subsection{Measurement method}

Firstly, we recorded a color video for a 26-year-old female participant. An overview of the general steps for HR measurement is illustrated in Figure 2. First, the measured region of interest (ROI) for any video image was localized, and here the forehead was selected to improve correction, as seen in Figure 2(a). The ROI was then decomposed into the three RGB channels, as shown in Figure 2(b), and 
spatially averaged over all pixels in the ROI to yield a red, blue and green measurement point for each frame at certain time and construct the raw traces $x_{1}(t), x_{2}(t)$ and $x_{3}(t)$ respectively, as shown in Figure $3(\mathrm{c})$. Subsequent processing was carried out for the whole $30 \mathrm{~s}$ video.

Secondly, to maximize the signals and minimize the noise, the weighted average of $x_{1}(t), x_{2}(t)$ and $x_{3}(t)$ can be calculated as the light intensity of the measured part according to the physiological information capacity in red, green and blue channel of color video recordings. It can be expressed as follows.

$$
I(t)=\left(\sum_{i=1}^{3} w_{i} x_{i}(t)\right) / 3 \quad(i=1,2,3)
$$

Here, $w_{i}$ is the weight of the raw RGB traces, and is decided by the attenuation degree of absorption and scatter of the tissue for red, green and blue light. So, the weight of $x_{2}(t)$ for green channel is the maximum, and that of $x_{3}(t)$ for blue channel is the minimum. Their numerical values vary less with the skin color, and are generally $0.59,0.3$ and 0.11 combining with optical properties of blood tissue.

Thus, we calculated the intensity component of the video frames, that is to say, the physical signal was obtained. The technique was called signal weighting analysis (SWA). The intensity signal was processed with the removal of baseline drift and de-noising using wavelet transform, and the filtered physical signal was obtained after the SWA was performed to the raw traces, as shown in Figure 2(d).

Finally, we obtained a power spectrum by applying FFT (Fast Fourier Transform) on the physical signal. And we designated the pulse frequency (heart rate) as the characteristic frequency corresponding to the greatest peak within a reliable frequency range. In our experiments, the reliable band was set at the interval of $[0.75,3] \mathrm{Hz}$ (corresponding to $[45,180] \mathrm{bpm}$ (beats per minute)) to keep a wider measurement range of heart rate.

Simultaneously, the reference values of heart rate were measured from the recorded electrical sphygmomanometer signal.

\subsection{Experiments}

An example of recovering human physiological parameters such as cardiac pulse rate obtained with a video recording of the female participant was given. According to the presented method, heart rate was measured correctly, as shown in Figure 3. We would not observe the cardiac pulse information in any of the red, green and blue noisy signals, but discussed the vital weighted physical signal emphatically. We discussed the superiority of the proposed SWA to the ICA and green-channel-only used to perform the pulse rate measurements in the experiment.

In the paper, we used a statistics index-reliability to describe the universal applicability of the proposed method. From 30 color facial video recordings from the 15 participants, the heart rates were calculated respectively to analyze the consistency of the measurements, as shown in Figure 4, and their statistics result can be obtained successfully.

\section{Results and discussion}

The three cardiovascular pulse waves, involving green-only trace, the signals respectively using ICA and SWA, were clearly visible and in Figure 3(a) and were closely consistent with the HR reference signal. The signal-to-noise ratios (SNR) of the three vital physical signals were calculated to 
be $48 \mathrm{~dB}, 33 \mathrm{~dB}$ and $29 \mathrm{~dB}$. In their power spectrum (Figure 3(b)), all clear peaks corresponding to the pulse rate were almost found at $1.3 \mathrm{~Hz}$, that is to say, the various times of the diffuse reflection light intensity on face in a minute was 78 averagely. The results indicate that heart rate can be obtained successfully through calculating the vital physical signals using SWA and ICA, or only analyzing the green trace. Compared those power spectrums, the propose SWA is better than ICA and green-channel only for heart rate measurement. The reference HR was calculated to be $1.25 \mathrm{~Hz}$, that is to say, the heart rate was $75 \mathrm{bpm}$. The error was calculated to be approximately $4 \%$.

The calculation results for 30 color videos were shown in Figure 4. The difference (HR $\left.\mathrm{HWA}_{\mathrm{S}}-\mathrm{HR}_{\mathrm{Ref}}\right)$ was less than $3 \mathrm{bpm}$ (Figure $4(\mathrm{a}))$, and the relative ratio $\left(\left(\mathrm{HR}_{\mathrm{SWA}}-\mathrm{HR}_{\mathrm{Ref}}\right) / \mathrm{HR}_{\mathrm{Ref}}\right)$ was less than $3.1 \%$. The root-mean-square error (RMSE) of the deviation for heart rate was $1.2 \mathrm{bpm}$, and their correlations coefficients $r$ was $0.94(p<0.001)$. The results indicated that the measurement approaches are valid and better for the younger.

It is possible that the physiological state can be seen using a CCD camera in ambient visible light. Compared with the traditional measurement techniques, like cardiac electrical conduction and cuffoscillometric measurements recognized as the common standard currently, which has the disadvantages of contact, physical discomfort, white coat effect, point-limited measurement, inaccuracy on large upper-arm circumferences, and so on, CCD diffuse reflection imaging measurement in this study has the characteristics of noncontact type, more comfortable sensation, noninterference, arbitrary body point and also reduces the white coat effect. Compared with the precious ICA and green channel only, the introduction of SWA not only improves the measurement accuracy of HR, but provides the possibility of BP measurement because the signal waveform obtained using SWA involves more cardiac pulse details. Besides, what needs illustrating is that the second physical signal in Figure 3(a) was obtained using ICA, referring to the Poh's proposed method [15]. Therefore, the work will have wider application fields.

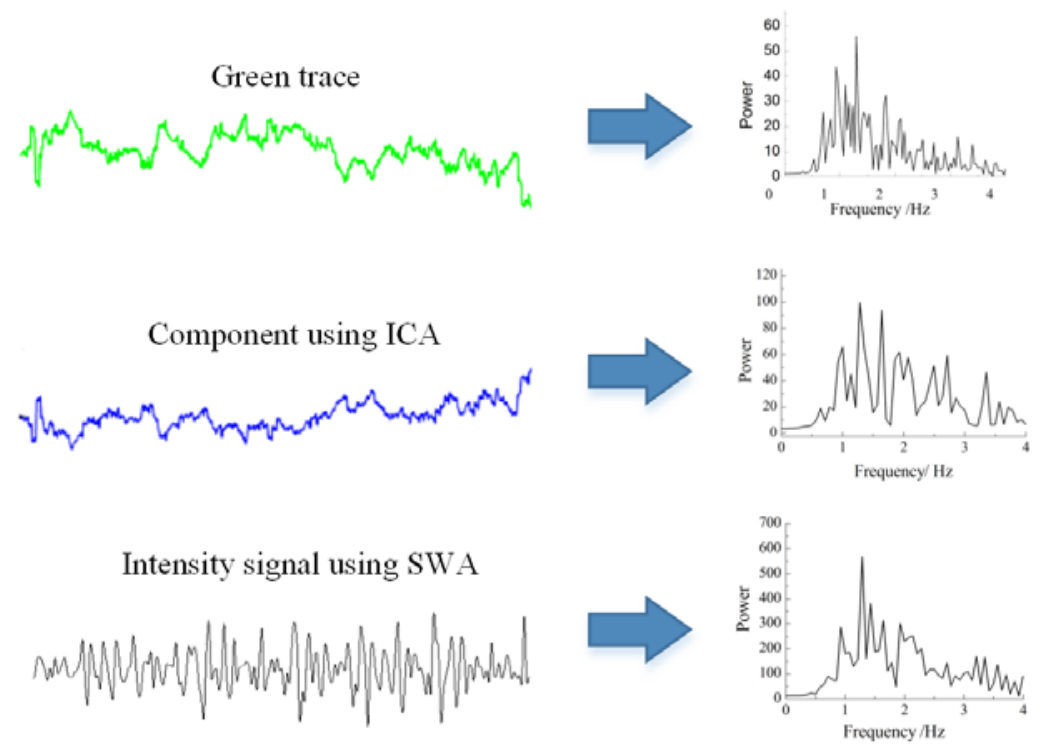

(a)

(b)

Fig. 3. Recovery of the cardiac pulse from the diffuse reflection light on the participant face. (a) 30s de-noised intensity signal; (b) power spectra. 


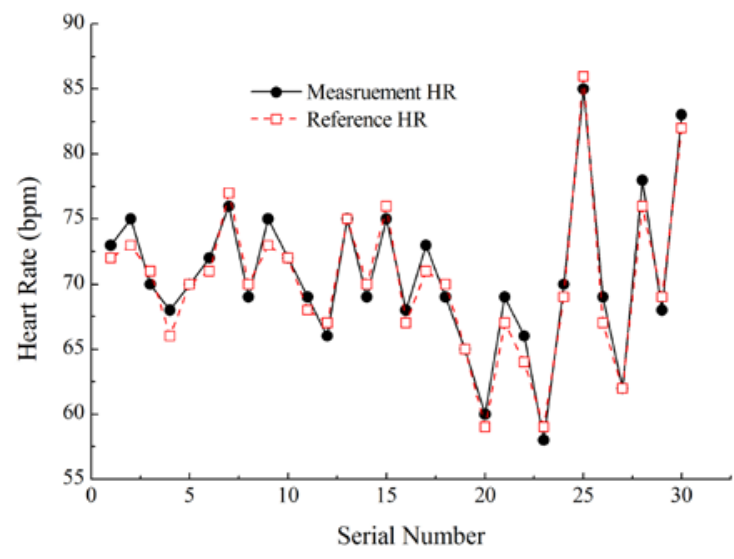

(a)

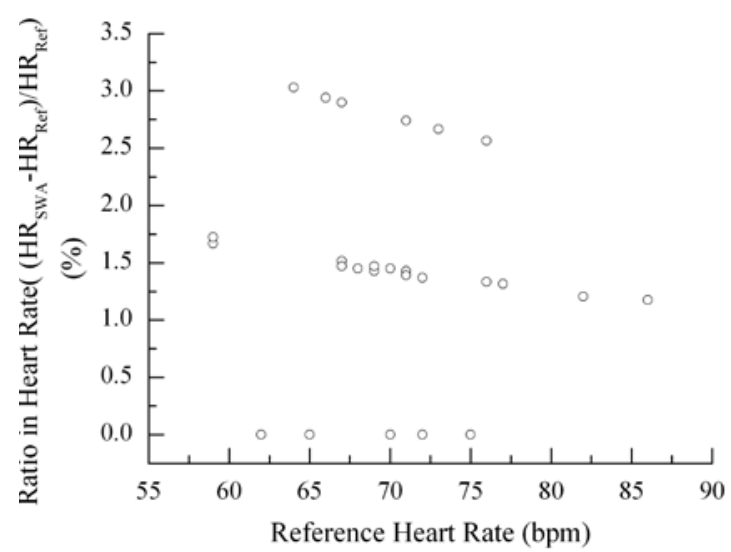

(b)

Fig. 4. Measurements for heart rate and its relative ratio.

Meanwhile, it must be admitted that there are limitations pertaining to the study. In Figure 3(a), the periodicity and waveform of the cardiac pulse wave using SWA were unobvious fully, which increased the difficulties and complications of the algorithm in computing the physiological parameters inevitably. The reason is possibly that the noncontact measurement technique, the unstable ambient light and the photoelectrical detection device with lower properties reduce the signal to noise ratio (SNR) of the intensity, which has greater effect on the measurement, although the signalweighted method was used. In the paper, we did not address that whether the algorithm is used to process the diffuse reflection light on the facial skins for different ages or not, but our experiments were conducted on the health and sick younger men in their twenties and thirties. The achievements of the study were not various significantly with the age range.

In further study, we will improve the intensity signal SNR to obtain high-accuracy results by taking the measures such as employing CCD camera with higher properties, controlling the illumination source and so on. Besides, we will not only discuss the possibility of BP measurement, but measure more samples to analyze the relationship of the optical parameters for different skins and the physiological parameters so as to expand the suitability and the applicable fields of the proposed method.

\section{Conclusion}

In the study, we have presented, implemented and assessed an improved method for measuring the physiological parameters like heart rate and respiratory rate from color videos recording the diffuse reflection intensity of human face ROI and illustrated an implementation with a CCD camera combining ambient plane illumination light source. The experimental results show that the imagingbased methods are suitable for measuring the physiological parameters with the analysis of human face complexions recorded by CCD camera in ambient light, and provide a reliable and comfortable measurement mode. It will be the subject of future study to create a real-time, wearable, multiparameter physiological monitor using this technique. 


\section{Acknowledgment}

This work is supported by the National Natural Science Foundation of China (NSFC, No. 61178040). The authors would like to express their sincere thanks to the NSFC and the reviewers for their insightful comments.

\section{References}

[1] D.D. Shao, Y.T. Yang, C.B Liu, et al., Noncontact monitoring breathing pattern, exhalation flow rate and pulse transit time, IEEE transactions on Biomedical Engineering 61 (2014), 2760-2767.

[2] S.S. Xie, H. Li, H.B. Niu, et al., Development and prospective of biomedical photonics, Scientia Sinica Physica, Mechanica \& Astronomica 37 (2007), 1-7.

[3] I.D. Stephen, M.J.L. Smith, M.R. Stirrat and D.I. Perrett, Facial skin coloration affects perceived health of human faces, International Journal of Primatology 30 (2009), 845-857.

[4] C. Scully, J.S. Lee, J. Meyer, et al., Physiological parameter monitoring from optical recordings with a mobile phone, IEEE Transactions on Biomedical Engineering 59 (2012), 303-306.

[5] H.Q. Yang, S.S. Xie, H. Li, et al., Determination of human skin optical properties in vivo from reflectance spectroscopic measurements, Chinese Optics Letters 5 (2007), 181-184.

[6] A. Zourabian, A. Siegel, B. Chance, et al., Trans-abdominal monitoring of fetal arterial blood oxygenation using pulse oximetry, Journal of Biomedical Optics 5 (2000), 391-405.

[7] A.B. Hertzman, The blood supply of various skin areas as estimated by the photoelectric plethysmograph, American Journal of Physiology 124 (1938), 328-340.

[8] J. Pickett, P. Amoroso, D.V. Nield, et al., Pulse oximetry and ppg measurements in plastic surgery, Proceedings of Annual International Conference on Engineering in Medicine \& Biology Society 5 (1997), 2330-2332.

[9] W. Verkruysse, L.O. Svaasand and J.S. Nelson, Remote plethysmographic imaging using ambient light, Optics Express 16 (2008), 21434-21445.

[10] C. Takano and Y. Ohta, Heart rate measurement based on a time-lapse image, Medical Engineering \& Physics 29 (2006), 853-857.

[11] M.Z. Poh, D.J. McDuff and R.W. Picard, Advancements in noncontact, multiparameter physiological measurements using a webcam, IEEE transactions on Biomedical Engineering 58 (2011), 7-11.

[12] F. Zhao, M. Li, Y. Qian and J.Z. Tsien, Remote measurements of heart and respiration rates for telemedicine, PLOS One 8 (2013), e71384.

[13] S.G. Demos, R. Gandour-Edwards, R. Ramsamooj and R. White, Spectroscopic detection of bladder cancer using nearinfrared imaging techniques, Journal of Biomedical Optics 9 (2004), 767-771.

[14] H. Wu, M. Rubinstein, E. Shih, J. Guttag, F. Durand and W. Freeman, Eulerian video magnification for revealing subtle changes in the world, ACM Transactions on Graphics 31 (2012), 65-72.

[15] M.Z. Poh, D.J. Mcduff and R.W. Picard, Non-contact, automated cardiac pulse measurements using video imaging and blind source separation, Optics Express 18 (2010), 10762.

[16] Yu Sun, Sijung Hu, Vicente Azorin-Peris, et al., Noncontact imaging photople-thysmography to effectively access pulse rate variability, Journal of Biomedical Optics 18 (2013), 061205.

[17] L.Q. Kong, Y.J. Zhao, L.Q. Dong, et al., Non-contact detection of oxygen saturation based on visible light imaging device using ambient light, Optics Express 21 (2013), 017464.

[18] Sheng Chieh Huang, Pei-Hsuan Hung, Chung-Hung Hong, et al., A new image blood pressure sensor based on PPG, RRT, BPTT, and harmonic balancing, IEEE sensors Journal 14 (2014), 3685-3692. 